Louisiana State University

LSU Digital Commons

Faculty Publications

Department of Chemistry

$10-11-2016$

\title{
Plasmon-Enhanced Photocleaving Dynamics in Colloidal MicroRNA-Functionalized Silver Nanoparticles Monitored with Second Harmonic Generation
}

\author{
Raju R. Kumal \\ Louisiana State University \\ Mohammad Abu-Laban \\ Louisiana State University \\ Corey R. Landry \\ Louisiana State University \\ Blake Kruger \\ Louisiana State University \\ Zhenyu Zhang \\ Louisiana State University
}

See next page for additional authors

Follow this and additional works at: https://digitalcommons.Isu.edu/chemistry_pubs

\section{Recommended Citation}

Kumal, R., Abu-Laban, M., Landry, C., Kruger, B., Zhang, Z., Hayes, D., \& Haber, L. (2016). PlasmonEnhanced Photocleaving Dynamics in Colloidal MicroRNA-Functionalized Silver Nanoparticles Monitored with Second Harmonic Generation. Langmuir, 32 (40), 10394-10401. https://doi.org/10.1021/ acs.langmuir.6b02538

This Article is brought to you for free and open access by the Department of Chemistry at LSU Digital Commons. It has been accepted for inclusion in Faculty Publications by an authorized administrator of LSU Digital Commons. For more information, please contact ir@lsu.edu. 


\section{Authors}

Raju R. Kumal, Mohammad Abu-Laban, Corey R. Landry, Blake Kruger, Zhenyu Zhang, Daniel J. Hayes, and Louis H. Haber 


\title{
Plasmon-Enhanced Photocleaving Dynamics in Colloidal MicroRNA-Functionalized Silver Nanoparticles Monitored with Second Harmonic Generation
}

\author{
Raju R. Kumal ${ }^{\dagger}$, Mohammad Abu-Laban $^{\ddagger}$, Corey R. Landry ${ }^{\ddagger}$, Blake Kruger $^{\dagger}$, Zhenyu \\ Zhang ${ }^{\dagger}$, Daniel J. Hayes§, and Louis H. Haber ${ }^{\dagger},{ }^{*}$ \\ tDepartment of Chemistry, Louisiana State University, Baton Rouge, Louisiana 70803, United \\ States \\ ‡Department of Biological and Agricultural Engineering, Louisiana State University, Baton Rouge, \\ Louisiana 70803, United States \\ §Department of Biomedical Engineering, the Pennsylvania State University, University Park, \\ Pennsylvania 16802, United States
}

\begin{abstract}
The photocleaving dynamics of colloidal micro-RNA-functionalized nanoparticles are studied using time-dependent second harmonic generation (SHG) measurements. Model drug-delivery systems composed of oligonucleotides attached to either silver nanoparticles or polystyrene nanoparticles using a nitrobenzyl photocleavable linker are prepared and characterized. The photoactivated controlled release is observed to be most efficient on resonance at $365 \mathrm{~nm}$ irradiation, with pseudo-first-order rate constants that are linearly proportional to irradiation powers. Additionally, silver nanoparticles show a 6-fold plasmon enhancement in photocleaving efficiency over corresponding polystyrene nanoparticle rates, while our previous measurements on gold nanoparticles show a 2-fold plasmon enhancement compared to polystyrene nanoparticles. Characterizations including extinction spectroscopy, electrophoretic mobility, and fluorimetry measurements confirm the analysis from the SHG results. The real-time SHG measurements are shown to be a highly sensitive method for investigating plasmon-enhanced photocleaving dynamics in model drug delivery systems.
\end{abstract}

\section{Graphical Abstract}

*Corresponding Author. 1haber@1su.edu. Phone: (225) 578-7965.

\section{ASSOCIATED CONTENT}

Supporting Information

The Supporting Information is available free of charge on the ACS Publications website at DOI: 10.1021/acs.langmuir.6b02538.

Details of the sequences and the photocleaving process, fluorescence signal of the SNPs before and after miRNA functionalization, measured SHG electric fields from miRNA-functionalized PSNPs as a function of irradiation time with $365 \mathrm{~nm}$ at different UV laser average powers, electrophoretic mobility of the miRNA-functionalized SNPs under different UV irradiation times, and $\zeta$ potential of the miRNA-functionalized SNPs under varying UV irradiation times (PDF)

The authors declare no competing financial interest. 

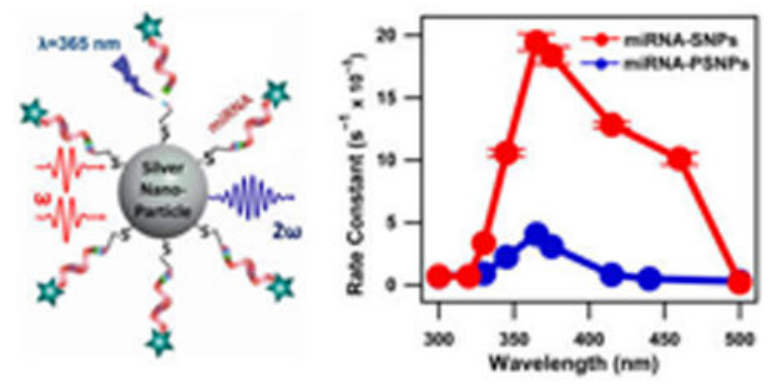

\section{INTRODUCTION}

Short nucleic acids such as microRNA (miRNA) and small interfering RNA (siRNA) play a significant role in posttranscriptional gene regulation, silencing, and profiling. ${ }^{1-3}$ These short RNA molecules are important in classifying and controlling tumor growth and cell differentiation, as well as other biological processes. ${ }^{4-6}$ In order to use miRNA or siRNA as a therapeutic, it is important that the oligonucleotides are protected before entering the bloodstream to avoid degradation. ${ }^{7-9}$ One approach for oligonucleotide protection involves modification with a bifunctional photolinker that connects to a colloidal metallic nanoparticle through a thiol bond. ${ }^{10,11}$ Photocleaving of the photolinker then releases the active oligonucleotide from the nanoparticle surface, leading to the corresponding regulation of gene expression. ${ }^{12}$ Although similar systems have been developed for programmed release, light activation is especially desirable because it provides a high degree of spatial and temporal control of miRNA delivery. ${ }^{10,13}$

Metallic nanoparticles such as gold nanoparticles (GNPs) and silver nanoparticles (SNPs) are promising for biological applications due to their biocompatibility, ease of synthesis, and convenient chemical functionalization with drug, oligonucleotide, and protein molecules. ${ }^{14-16}$ Gold and silver nanoparticles have surface plasmon resonances characterized by the coherent oscillation of free electrons upon interaction with incident light leading to the enhancement of optical scattering and absorption processes. ${ }^{17-21}$ These nanoparticles are also ideally suited for contrasting agents, cellular imaging, and exothermic reactors. ${ }^{22,23}$ Additionally, silver nanoparticles have wound healing antimicrobial activities, ${ }^{24}$ demonstrate negligible cytotoxicities in small doses, ${ }^{25,26}$ and can be utilized for drug-delivery applications in photoactivated gene silencing. ${ }^{27,28}$ Plasmonic release of oligonucleotides has been studied from the surface of colloidal gold nanoparticles. ${ }^{29-32}$ Previously, we have investigated the kinetics of photoactivated controlled-release of miRNA from the surface of colloidal gold nanoparticles using time-resolved second harmonic generation $(\mathrm{SHG}){ }^{33}$

Nonlinear optical spectroscopy is a powerful surface-sensitive method for probing phenomena in biology and nanotechnology. ${ }^{34-40}$ Second harmonic generation is a nonlinear spectroscopy that is especially useful for probing the surface of colloidal nanoparticles. ${ }^{41-49}$ In SHG, two incident photons of frequency $\omega$ combine to produce a photon of frequency $2 \omega$. Second harmonic generation is dipole forbidden in centrosymmetric bulk media, but it can be generated from the colloidal nanoparticle surface where the inversion symmetry is 
broken. The SHG signal from a charged interface has contributions from the second-order and third-order nonlinear susceptibilities, $\chi^{(2)}$ and $\chi^{(3)}$, respectively. The $\chi^{(2)}$ term originates from the surface-specific two-photon spectroscopy of the nanoparticle, while the $\chi^{(3)}$ term depends upon the polarization of the bulk solvent molecules induced by the electric field of the nanoparticle surface. The SHG electric field, $E_{\mathrm{SHG}}$, is proportional to the square root of the intensity of the SHG signal, with ${ }^{50-58}$

$$
E_{\mathrm{SHG}}=\chi^{(2)} E_{\omega} E_{\omega}+\chi^{(3)} E_{\omega} E_{\omega} \phi_{0}
$$

where $E_{\omega}$ is the electric field of the incident laser beam with frequency $\omega$ and $\phi_{0}$ is the electrostatic surface potential of the nanoparticle.

In this paper, the efficiency of light-activated release of oligonucleotides from the surface of silver, gold, and polystyrene nanoparticles are compared by using SHG spectroscopy. The size of the nanoparticles are all roughly $65 \mathrm{~nm}$ diameter, and they are functionalized with miRNA-148b and a nitrobenzyl photocleaveable (PC) linker. Additional information on the miRNA functionalization of the colloidal nanoparticles is described in the Supporting Information. The functionalized nanoparticles are irradiated with varying laser powers and wavelengths and are simultaneously probed by SHG from $800 \mathrm{~nm}$ laser pulses to study the kinetics of the photocleaving process. The probe laser itself shows no photoreleasing effect on the sample. Careful analysis of the power and wavelength dependent data shows that the photolysis is a one-photon process. By comparing the releasing rates of oligonucleotides from three different nanoparticles, it is found that the photolysis is enhanced by the plasmon of the silver nanoparticle. The SHG studies at different laser powers and wavelengths are directly compared to corresponding fluorescence quantification measurements and extinction spectra for each sample and experimental condition.

\section{SYNTHESIS AND CHARACTERIZATION OF NANOPARTICLES}

The colloidal silver nanoparticles are prepared in water by reduction of silver nitrate using citrate and ascorbic acid in the presence of potassium iodide, ${ }^{59}$ followed by miRNA functionalization. Briefly, an aqueous solution of $2.54 \mathrm{~mL}$ containing $5.91 \mathrm{mM}$ silver nitrate, $13.4 \mathrm{mM}$ sodium citrate, and $1.54 \mu \mathrm{M}$ potassium iodide is added to $47.5 \mathrm{~mL}$ of $210 \mu \mathrm{M}$ ascorbic acid in nanopure water under boiling and vigorous stirring conditions. The solution is boiled for an hour during which the color changes from colorless to light yellow resulting in relatively monodisperse, spherical, colloidal silver nanoparticles. miRNA is functionalized to the surface of nanoparticles via a "salt-aging" technique as described previously, ${ }^{10,33}$ where $2.5 \mu \mathrm{L}$ of $0.8 \mu \mathrm{g} / \mu \mathrm{L}$ miRNA (PC-miR-148b) labeled with a 6TAMRA fluorophore group and modified with a thiolized photocleaveable spacer is added to $1 \mathrm{~mL}$ of the silver nanoparticle solution. The samples are then left to incubate under gentle agitation conditions for a $24 \mathrm{~h}$ period. After incubation, $10 \mu \mathrm{L}$ of $0.035 \mathrm{M}$ sodium dodecyl sulfate (SDS) solution and $20 \mu \mathrm{L}$ of $0.01 \mathrm{M}$ phosphate-buffered saline (PBS) solution are added to each sample of colloidal nanoparticles, followed by an additional $3 \mathrm{~h}$ of incubation. Afterward, three rounds of the addition of $10 \mu \mathrm{L}$ of $2 \mathrm{M}$ Tris-sodium chloride solution with 
$4 \mathrm{~h}$ of incubation between each salting step is applied. The miRNA-functionalized nanoparticles are purified via centrifugation and finally resuspended in nanopure water.

For the polystyrene sulfate nanoparticles (PSNPs), a thiol-amine cross-linker succinimidyl 4-( $N$-maleimidomethyl) cyclohexane-1-carboxylate (SMCC) is used to functionalize the thiolated oligo to the nanoparticle surface. ${ }^{60} \mathrm{Here}, 70 \mu \mathrm{L}$ of $1 \mathrm{mg} / \mathrm{mL}$ SMCC in dimethyl sulfoxide solvent is added to $1 \mathrm{~mL}$ of the $1 \%$ amine-functionalized $60 \pm 5 \mathrm{~nm}$ PSNP aqueous solution. The SMCC-PSNP mixture is allowed to rock overnight at room temperature, then is centrifuged and resuspended in nanopure water before adding the miRNA. The same procedure for the miRNA functionalization as described above is then followed for the PSNP sample.

The nanoparticles are characterized using dynamic light scattering (DLS), transmission electron microscopy (TEM), $\zeta$-potential, fluorimetry, and extinction spectroscopy measurements. Representative TEM images of the silver nanoparticles before and after miRNA functionalization are shown in Figure 1a,b, respectively. From a survey of nanoparticle images, the average nanoparticle diameter is determined to be $65 \pm 7.5 \mathrm{~nm}$. Using dynamic light scattering, the hydrodynamic diameter of the SNPs before miRNA functionalization is measured to be $66 \pm 18 \mathrm{~nm}$, and the hydrodynamic diameter increases to $127 \pm 25 \mathrm{~nm}$ after miRNA functionalization. Figure 2 shows the experimental extinction spectrum of the colloidal silver nanoparticles (red solid line) when compared with a Mie theory fit (black dotted line) using a concentration of $9.55 \times 10^{9}$ nanoparticles $/ \mathrm{mL}$. The localized surface plasmon peak for silver nanoparticles is observed at $424 \mathrm{~nm}$. Figure 3 displays the electrophoretic mobility of nanoparticles before and after miRNA functionalization. The electrophoretic mobility of the SNPs is measured to be $(-2.60 \pm 0.53)$ $\times 10^{-8} \mathrm{~m}^{2} /(\mathrm{V} \mathrm{s})$ with a corresponding $\zeta$ potential of $-49.80 \pm 10.17 \mathrm{mV}$ using Huckel's approximation. ${ }^{33,61}$ The electrophoretic mobility of the miRNA-functionalized SNPs is measured to be $(-3.86 \pm 0.60) \times 10^{-8} \mathrm{~m}^{2} /(\mathrm{V} \mathrm{s})$ with a corresponding $\zeta$-potential of -74.80 $\pm 11.40 \mathrm{mV}$. Additional characterizations are shown in the Supporting Information.

\section{EXPERIMENTAL SETUP}

The controlled release of miRNA from the surface of colloidal nanoparticles is performed using an experimental setup, which has been previously described. ${ }^{39}$ Briefly, the experimental setup consists of an ultrafast laser system, an optical setup, and a high sensitive charged-coupled device (CCD) detector connected to a monochromator. A titanium:sapphire oscillator laser with $70 \mathrm{fs}$ pulses centered at $800 \mathrm{~nm}$ at an $80 \mathrm{MHz}$ repetition rate and an average power of $2.6 \mathrm{~W}$ is used. A portion of the oscillator beam passes through a beam splitter to seed the amplifier laser, and the remaining portion is used for the SHG measurements. An amplified laser ( $75 \mathrm{fs}, 800 \mathrm{~nm}, 10 \mathrm{kHz}, 7 \mathrm{~W}$ ) pumps an optical parametric amplifier (OPA) to generate the wavelength-tunable photocleaving irradiation beam with $100 \mathrm{fs}$ pulses at a $10 \mathrm{kHz}$ repetition rate. For SHG measurements, the $800 \mathrm{~nm}$ probe laser from the oscillator is attenuated to an average power of $790 \mathrm{~mW}$ before being focused to a $1 \mathrm{~cm} \times 1 \mathrm{~cm}$ quartz cuvette containing the sample at a concentration of $8.3 \times$ $10^{9}$ nanoparticles $/ \mathrm{mL}$. The photocleaving irradiation beam at the desired wavelength and power is directed to the sample at $90^{\circ}$ with respect to the SHG probe laser. The SHG from 
the nanoparticle sample is measured as a function of time using different photocleaving irradiation wavelengths and average powers. An integrated Labview program controls a magnetic stir bar and a beam block to open and shut every $30 \mathrm{~s}$ in synchronization with automated data acquisition for background-subtracted time-dependent SHG measurements.

\section{RESULTS AND DISCUSSION}

The photocleaving controlled release of miRNA from the surface of silver nanoparticles and polystyrene nanoparticles is measured in real time using second harmonic generation. The obtained results are compared with our previous studies on miRNA-functionalized gold nanoparticles. ${ }^{33}$ Representative SHG spectra of SNPs and miRNA-functionalized SNPs are shown in Figure 4a. The SHG peak from the $800 \mathrm{~nm}$ probe laser is located at $400 \mathrm{~nm}$, as expected, with full width half-maximum of $4.5 \mathrm{~nm}$. The small signal at longer wavelengths is due to the two-photon fluorescence from the SNPs. The SHG signal intensity from miRNA-functionalized SNPs is approximately four times higher than the corresponding SHG signal from the SNPs using the same laser conditions and nanoparticle concentrations. The higher SHG signal in the miRNA-functionalized SNPs is mainly attributed to the negatively charged phosphate groups of the oligonucleotides, which increase the surface charge density magnitude and the corresponding surface potential, resulting in a larger $\chi^{(3)}$ term. This interpretation is consistent with previous work and with our earlier results on miRNA-functionalized GNPs. ${ }^{10,33,58,62}$ The 6-TAMRA fluorophore has negligible contribution to the SHG signal because it has negligible absorption near the $800 \mathrm{~nm}$ probe and $400 \mathrm{~nm}$ SHG wavelengths. Figure $4 \mathrm{~b}$ shows representative SHG spectra from the miRNA-functionalized SNPs at different UV-irradiation times of 0, 90, and $800 \mathrm{~s}$ using 365 $\mathrm{nm}$ irradiation with a $20 \mathrm{~mW}$ average power. The decrease in SHG intensity under increasing UV-irradiation time is due to the photocleaving of miRNA from the colloidal SNP surface, resulting in a decrease of the nanoparticle surface charge density magnitude and a smaller $\chi^{(3)}$ term.

The results from control experiments are shown in Figure 5a where the variation of the SHG intensity as a function of the UV-irradiation time is displayed for the SNPs and the miRNAfunctionalized SNPs. The SHG signal from the miRNA-functionalized SNP sample remains constant over time using the $800 \mathrm{~nm}$ probe laser, indicating that no photocleaving occurs from the probe laser alone. Similarly, the SHG signal from the SNP sample remains constant over time when both the $800 \mathrm{~nm}$ probe laser and the UV laser at $365 \mathrm{~nm}$ with an average power of $20 \mathrm{~mW}$ are used. However, the SHG intensity from the miRNA-functionalized SNP sample decreases and asymptotically reaches a minimum value when using both the probe laser and the UV laser. The minimum of the time-dependent SHG signal from the miRNA-functionalized under UV irradiation is equal to the corresponding signal of the SNPs, indicating complete photocleaving of oligonucleotides from the surface of nanoparticles.

A wavelength-dependent study on the photocleaving process is shown in Figure 5b. The error bars are included in Figure 5a,b but are mostly smaller than the data points. The irradiation laser wavelength is varied from 300 to $500 \mathrm{~nm}$, and time-dependence of the SHG signal is measured to determine the photocleaving rate under a constant average irradiation 
laser power of $20 \mathrm{~mW}$. The fastest decay of the SHG signal is observed at $365 \mathrm{~nm}$ irradiation, in agreement with previous measurements on miRNA-functionalized gold nanoparticles, ${ }^{33}$ indicating the photocleaving peak resonance. Relatively rapid photocleaving kinetics are also observed at irradiation wavelengths between 345 and $460 \mathrm{~nm}$. Much slower photocleaving is observed using irradiation at $330 \mathrm{~nm}$, while almost no photocleaving is observed with irradiation at 300,320 , and $500 \mathrm{~nm}$, which are far from the photocleaving resonance.

The wavelength-dependent photocleaving rates in the plasmonic miRNA-functionalized SNPs are compared with corresponding measurements in the nonplasmonic miRNAfunctionalized polystyrene nanoparticles in order to determine the role of plasmonic enhancement of the photocleaving process. The time-dependent SHG signals from the miRNA-functionalized PSNPs are shown in Figure 6 under varying irradiation wavelengths from 300 to $500 \mathrm{~nm}$ at $20 \mathrm{~mW}$. The photocleaving rates from the miRNA-functionalized PSNPs are lower than the corresponding photocleaving rates from the SNP sample.

The photocleaving rate constants at different irradiation wavelengths are determined for the miRNA-functionalized SNPs and PSNPs by fitting the SHG data using a pseudo-first-order exponential rate equation, as described previously. ${ }^{33}$ The SHG electric field can be described by the equation $E_{\mathrm{SHG}}=A+B[C]$, where $A$ is an offset, $B$ is a proportionality constant, and $[C]$ is the cumulative concentration of miRNA attached to the surface of nanoparticles. As noted earlier, the change in $E_{\mathrm{SHG}}$ as a function of attached miRNA at the nanoparticle surface is attributed mainly to the surface charge density and the resulting $\chi^{(3)}$ term. ${ }^{33,58,62}$ Under the UV irradiation-induced photocleaving, the attached miRNA concentration should exponentially decay in time $t$ according to the equation, $[C](t)=[C]_{0} \mathrm{e}^{-k^{\prime} t}$, where $[C]_{0}$ is the initial concentration of miRNA attached to the nanoparticle surface and the pseudo-firstorder rate constant $k^{\prime}$ is linearly proportional to the UV laser intensity, assuming that the photocleaving is a single-photon process.

The resulting rate constants for the miRNA-functionalized SNP and PSNP samples determined from the pseudo-first-order fits are plotted as a function of the irradiation wavelength in Figure 7. Both miRNA-functionalized nanoparticles show a maximum rate of photocleaving at $365 \mathrm{~nm}$ due to the characteristic resonance peak of the photocleaving process. The miRNA-functionalized SNPs show greatly enhanced photocleaving rate constants compared to the PSNPs over the irradiation wavelengths from 350 to $450 \mathrm{~nm}$ due to plasmon enhancement. The plasmon resonance of the SNPs cause enhanced optical fields near the nanoparticle surface leading to plasmon-enhanced photocleaving over the wavelength range of the SNP plasmon resonance, which directly overlaps with the photocleaving resonance of the nitrobenzyl linker. The PSNP sample has no associated plasmon resonance, so its rate constants are attributed to the nitrobenzyl linker alone.

The power-dependent photocleaving of the miRNA-functionalized SNPs and PSNPs is also studied for additional insight. Figure 8a shows the SHG electric field from miRNAfunctionalized SNPs as a function of UV irradiation time using $365 \mathrm{~nm}$ at different UV laser powers. The rate constants from the miRNA-functionalized SNPs obtained at the laser powers of $15,25,35,45,55$, and $75 \mathrm{~mW}$ are $(4.9 \pm 0.1) \times 10^{-3},(8.6 \pm 0.2) \times 10^{-3},(12.4$ 
$\pm 0.6) \times 10^{-3},(16.1 \pm 0.3) \times 10^{-3},(18.0 \pm 0.2) \times 10^{-3}$, and $(26.3 \pm 0.6) \times 10^{-3} \mathrm{~s}^{-1}$, respectively. Corresponding results from the miRNA-functionalized PSNPs are shown in the Supporting Information. The photocleaving rate constants for the miRNA-functionalized SNPs and PSNPs are plotted as a function of the UV laser average powers in Figure 8b. The error bars are included in Figure 8a,b but are mostly smaller than the data points. The previously obtained GNP data ${ }^{33}$ are included for direct comparison. The linear variation of the rate constants as a function of the UV laser power is consistent with a pseudo-first-order kinetics model, indicating that the photocleaving is a one-photon process for all nanoparticle samples investigated. The slopes obtained for miRNA-functionalized SNPs and PSNPs are $(3.48 \pm 0.12) \times 10^{-4} \mathrm{~s}^{-1} \mathrm{~mW}^{-1}$ and $(5.45 \pm 0.28) \times 10^{-5} \mathrm{~s}^{-1} \mathrm{~mW}^{-1}$, respectively, with corresponding $y$-intercepts of $(1.3 \pm 6.0) \times 10^{-4} \mathrm{~s}^{-1}$ and $(0.2 \pm 1.4) \times 10^{-4} \mathrm{~s}^{-1}$, respectively. Here, the $y$-intercepts are equal to zero to within experimental uncertainty, in agreement with the observation that no photocleaving occurs without UV irradiation. The ratios of the slopes of the change in the rate constants under changing UV laser powers for the SNPs and GNPs compared to the PSNPs provide a direct method for determining the plasmon enhancement factors. Using this method, the plasmon enhancement factors for photocleaving in the SNPs and GNPs are $6.4 \pm 0.4$ and $2.3 \pm 0.2$, respectively. The SNP enhancement is much higher because its plasmon resonance directly overlaps with the photocleaving resonance wavelength of the nitrobenzyl linker. The GNP plasmon is centered near $540 \mathrm{~nm}$, and its extinction near $365 \mathrm{~nm}$ is dominated by the electronic interband transition, ${ }^{45,63}$ causing a decrease in the relative optical field enhancement.

Additional characterization measurements are performed to confirm the analysis obtained from the SHG results. Figure 9 shows the extinction spectra of the SNPs, the miRNAfunctionalized SNPs, and the miRNA-functionalized SNPs after laser irradiation with 330, 365 , and $435 \mathrm{~nm}$ at an average power of $20 \mathrm{~mW}$ for $14 \mathrm{~min}$. The plasmon peak of the SNP sample shifts from 424 to $435 \mathrm{~nm}$ after functionalization with miRNA due to the change in the dielectric constant at the nanoparticle surface. The plasmon peak of the miRNAfunctionalized SNPs blue shifts back toward the SNP spectrum under UV laser irradiation. After $365 \mathrm{~nm}$ irradiation, the miRNA-functionalized SNPs plasmon spectrum closely matches the SNP spectrum, indicating complete photocleaving. The miRNA-functionalized SNP spectra deviate from the SNP after 330 and $435 \mathrm{~nm}$ irradiation, indicating incomplete photocleaving.

Quantification of the miRNA released from the nanoparticle surface is obtained from fluorescence measurements of the 6-TAMRA labeling fluorophore in the centrifuged supernatants following laser irradiation or chemical reduction according to the procedure described previously. ${ }^{33}$ Figure 10 shows the fluorescent signal from the miRNAfunctionalized SNPs after 14 min irradiation with $20 \mathrm{~mW}$ average power for wavelengths ranging from 300 to $800 \mathrm{~nm}$. These measurements are compared to the supernatants after the addition of dithiothreitol (DTT) for compete reduction and chemical detachment of the miRNA. Irradiation with $365 \mathrm{~nm}$ shows maximum photocleaving corresponding to complete release, to within experimental uncertainty. Irradiation at other wavelengths show partial photocleaving, with negligible photocleaving at 300 and $800 \mathrm{~nm}$, in agreement with the SHG measurements. The concentration of the SNPs is determined by inductively coupled plasma optical emission spectroscopy (ICP-OES). When combined with the fluorimetry 
measurements, the average oligonucleotide surface densities are determined to be (5.92 $\pm 0.76) \times 10^{-3}$ and $(6.18 \pm 0.45) \times 10^{-3}$ oligo/nm ${ }^{2}$ for the SNPs and PSNPs, respectively, corresponding to coverages of $79 \pm 10$ oligo/nanoparticle and $70 \pm 5$ oligo/nanoparticle, respectively.

\section{CONCLUSION}

The photocleaving dynamics from model oligonucleotide therapeutic delivery systems are studied using time-dependent second harmonic generation measurements. The photocleaving rates from the surface of colloidal miRNA-functionalized silver nanoparticles and polystyrene nanoparticles in water are obtained using different laser irradiation wavelengths and powers. The photocleaving rate is maximized using the irradiation wavelength of $365 \mathrm{~nm}$, which is on resonance with the nitrobenzyl linker. A linear variation of the photocleaving rate constant under varying irradiation laser powers is observed, demonstrating that the associated kinetics are described by a one-photon process. The miRNA-functionalized silver nanoparticles show a plasmon enhancement factor of $6.4 \pm 0.4$ when compared to the photocleaving rates from corresponding measurements in the polystyrene nanoparticles. Electrophoretic mobility, extinction spectroscopy, and fluorimetry measurements are used to verify the analysis of the SHG results, which are compared to previous investigations on gold nanoparticles. Second harmonic generation is shown to be a very sensitive nonlinear optical technique for investigating real-time plasmonenhanced photocleaving for potential drug-delivery systems.

\section{MATERIALS AND METHODS}

\section{Chemicals and Reagents}

For silver nanoparticle synthesis, silver nitrate ( $\geq 99 \%$ ), sodium citrate dihydrate ( $\geq 99 \%$ ), potassium iodide ( $\geq 99 \%$ ), phosphate buffer saline solution (PBS), sodium chloride ( $\geq 98 \%$ ), anhydrous ethanol, and dithiothreitol (1 M) were purchased from Sigma-Aldrich, and ascorbic acid ( $\geq 99 \%$ ) was purchased from Sigma Life Science. Custom modified miRNA-148b was received from Trilink Bio Technologies. Diethylpyrocarbonate water was purchased from Ambion, sodium dodecyl sulfate ( $\ 99 \%$ ) from VWR, and tris(hydroxymethyl) aminomethane ( $299.9 \%$ ) solid from Amresco. The $1 \%$ polystyrene nanoparticle solution with amine functionalization was purchased from Nanocs, and succinimidyl 4-( $N$-maleimidomethyl) cyclohexane-1-carboxylate ( $\$ 99 \%)$ was received from Thermo Fisher. Nanopure water with resistivity greater than $18.2 \mathrm{M} \Omega$ was used as a solvent during the synthesis of the miRNA-functionalized nanoparticles, as well as for spectroscopic measurements.

\section{Characterization of Nanoparticles}

S-Potential and dynamic light scattering measurements were taken by Zetasizer Nano ZS from Malvern Instruments Inc., UK. Fluorescence measurements were performed using a Wallac VICTOR2 V1420-040 Multilabel Counter fluorimeter and extinction measurements were done using UV/vis spectrometer from PerkinElmer, Boston, USA. The TEM images were obtained using a high resolution JEOL JEM-2011 with a Gatan SC1000 CCD camera 
and an EDAX EDS. The ICP-OES measurement was performed to quantify the nanoparticles using a Varian Vista-MPX, Palo Alto, CA, USA.

\section{Supplementary Material}

Refer to Web version on PubMed Central for supplementary material.

\section{Acknowledgments}

The authors would like to thank Louisiana State University, the National Science Foundation (CBET-1254281), and the National Institute Dental and Craniofacial Research of the National Institutes of Health (RDE024790A) for generous financial support. The content is solely the responsibility of the authors and does not necessarily represent the official views of the National Institutes of Health. Caroline Copeland helped with the TOC graphic. The authors also acknowledge to Dr. Rafael Cueto for helping in dynamic light scattering and zeta potential measurements and LSU Material Characterization Center (LSUMCC) for transmission electron microscopy images.

\section{REFERENCES}

1. Bartel DP. MicroRNAs: Genomics, Biogenesis, Mechanism, and Function. Cell. 2004; 116:281297. [PubMed: 14744438]

2. Lu J, Getz G, Miska EA, Alvarez-Saavedra E, Lamb J, Peck D, Sweet-Cordero A, Ebert BL, Mak RH, Ferrando AA, et al. MicroRNA Expression Profiles Classify Human Cancers. Nature. 2005; 435:834-838. [PubMed: 15944708]

3. Macha MA, Seshacharyulu P, Krishn SR, Pai P, Rachagani S, Jain M, Batra SK. MicroRNAs (miRNAs) as Biomarker (s) for Prognosis and Diagnosis of Gastrointestinal (GI) Cancers. Curr. Pharm. Des. 2014; 20:5287-5297. [PubMed: 24479799]

4. Greene SB, Herschkowitz JI, Rosen JM. Small Players With Big Roles: MicroRNAs as Targets to Inhibit Breast Cancer Progression. Curr. Drug Targets. 2010; 11:1059-1073. [PubMed: 20545613]

5. Reynolds A, Leake D, Boese Q, Scaringe S, Marshall WS, Khvorova A. Rational siRNA Design for RNA Interference. Nat. Biotechnol. 2004; 22:326-330. [PubMed: 14758366]

6. Zhang B, Pan X, Cobb GP, Anderson TA. MicroRNAs as Oncogenes and Tumor Suppressors. Dev. Biol. 2007; 302:1-12. [PubMed: 16989803]

7. Muro S. Challenges in Design and Characterization of Ligand-Targeted Drug Delivery Systems. J. Controlled Release. 2012; 164:125-137.

8. Verma A, Uzun O, Hu Y, Hu Y, Han H-S, Watson N, Chen S, Irvine DJ, Stellacci F. SurfaceStructure-Regulated Cell-Membrane Penetration by Monolayer-Protected Nanoparticles. Nat. Mater. 2013; 12:376-376.

9. Zhou J, Wu J, Hafdi N, Behr J-P, Erbacher P, Peng L. PAMAM Dendrimers for Efficient siRNA Delivery and Potent Gene Silencing. Chem. Commun. 2006; 22:2362-2364.

10. Qureshi AT, Monroe WT, Dasa V, Gimble JM, Hayes DJ. miR-148b-Nanoparticle Conjugates for Light Mediated Osteogenesis of Human Adipose Stromal/Stem Cells. Biomaterials. 2013; 34:7799-7810. [PubMed: 23870854]

11. Zhang J, Malicka J, Gryczynski I, Lakowicz JR. Surface-Enhanced Fluorescence of FluoresceinLabeled Oligonucleotides Capped on Silver Nanoparticles. J. Phys. Chem. B. 2005; 109:76437648. [PubMed: 16851886]

12. Vivero-Escoto JL, Slowing II, Wu C-W, Lin VS-Y. Photoinduced Intracellular Controlled Release Drug Delivery in Human Cells by Gold-Capped Mesoporous Silica Nanosphere. J. Am. Chem. Soc. 2009; 131:3462-3463. [PubMed: 19275256]

13. Brown PK, Qureshi AT, Moll AN, Hayes DJ, Monroe WT. Silver Nanoscale Antisense Drug Delivery System for Photoactivated Gene Silencing. ACS Nano. 2013; 7:2948-2959. [PubMed: 23473419]

14. Thakor AS, Gambhir SS. Nanooncology: the Future of Cancer Diagnosis and Therapy. Ca-Cancer J. Clin. 2013; 63:395-418. [PubMed: 24114523] 
15. Couvreur P. Nanoparticles in drug delivery: past, present and future. Adv. Drug Delivery Rev. 2013; 65:21-23.

16. Dreaden EC, Alkilany AM, Huang X, Murphy CJ, El-Sayed MA. The Golden Age: Gold Nanoparticles for Biomedicine. Chem. Soc. Rev. 2012; 41:2740-2779. [PubMed: 22109657]

17. Thomas KG, Kamat PV. Chromophore-Functionalized Gold Nanoparticles. Acc. Chem. Res. 2003; 36:888-898. [PubMed: 14674780]

18. Jain PK, Lee KS, El-Sayed IH, El-Sayed MA. Calculated Absorption and Scattering Properties of Gold Nanoparticles of Different Size, Shape, and Composition: Applications in Biological Imaging and Biomedicine. J. Phys. Chem. B. 2006; 110:7238-7248. [PubMed: 16599493]

19. Kelly KL, Coronado E, Zhao LL, Schatz GC. The Optical Properties of Metal Nanoparticles: the Influence of Size, Shape, and Dielectric Environment. J. Phys. Chem. B. 2003; 107:668-677.

20. Yang X, Yang M, Pang B, Vara M, Xia Y. Gold Nanomaterials at Work in Biomedicine. Chem. Rev. 2015; 115:10410-10488. [PubMed: 26293344]

21. Yguerabide J, Yguerabide EE. Light-Scattering Submicroscopic Particles as Highly Fluorescent Analogs and Their Use as Tracer Labels in Clinical and Biological Applications: I. Theory. Anal. Biochem. 1998; 262:137-156. [PubMed: 9750128]

22. Hong R, Han G, Fernández JM, Kim B-j, Forbes NS, Rotello VM. Glutathione-Mediated Delivery and Release Using Monolayer Protected Nanoparticle Carriers. J. Am. Chem. Soc. 2006; 128:1078-1079. [PubMed: 16433515]

23. You J, Zhang G, Li C. Exceptionally High Payload of Doxorubicin in Hollow Gold Nanospheres for Near-Infrared Light-Triggered Drug Release. ACS Nano. 2010; 4:1033-1041. [PubMed: 20121065]

24. Kim JS, Kuk E, Yu KN, Kim J-H, Park SJ, Lee HJ, Kim SH, Park YK, Park YH, Hwang C-Y, et al. Antimicrobial Effects of Silver Nanoparticles. Nanomedicine. 2007; 3:95-101. [PubMed: 17379174]

25. Kim YS, Kim JS, Cho HS, Rha DS, Kim JM, Park JD, Choi BS, Lim R, Chang HK, Chung YH, et al. Twenty-Eight-Day Oral Toxicity, Genotoxicity, and Gender-Related Tissue Distribution of Silver Nanoparticles in Sprague-Dawley Rats. Inhalation Toxicol. 2008; 20:575-583.

26. Tiwari DK, Jin T, Behari J. Dose-Dependent In-Vivo Toxicity Assessment of Silver Nanoparticle in Wistar Rats. Toxicol. Mech. Methods. 2011; 21:13-24. [PubMed: 21080782]

27. Qureshi AT, Monroe WT, Lopez MJ, Janes ME, Dasa V, Park S, Amirsadeghi A, Hayes DJ. Biocompatible/Bioabsorbable Silver Nanocomposite Coatings. J. Appl. Polym. Sci. 2011; 120:3042-3053.

28. Qureshi AT, Terrell L, Monroe WT, Dasa V, Janes ME, Gimble JM, Hayes DJ. Antimicrobial Biocompatible Bioscaffolds for Orthopaedic Implants. J. Tissue Eng. Regener. Med. 2014; 8:386395.

29. Simoncelli S, de Alwis Weerasekera H, Fasciani C, Boddy CN, Aramendia PF, Alarcon EI, Scaiano JC. Thermoplasmonic ssDNA Dynamic Release from Gold Nanoparticles Examined with Advanced Fluorescence Microscopy. J. Phys. Chem. Lett. 2015; 6:1499-1503. [PubMed: 26263158]

30. Poon L, Zandberg W, Hsiao D, Erno Z, Sen D, Gates BD, Branda NR. Photothermal Release of Single-Stranded DNA From the Surface of Gold Nanoparticles Through Controlled Denaturating and Au-S Bond Breaking. ACS Nano. 2010; 4:6395-6403. [PubMed: 20958080]

31. Huschka R, Neumann O, Barhoumi A, Halas NJ. Visualizing Light-Triggered Release of Molecules Inside Living Cells. Nano Lett. 2010; 10:4117-4122. [PubMed: 20857946]

32. Thibaudau F. Ultrafast Photothermal Release of DNA From Gold Nanoparticles. J. Phys. Chem. Lett. 2012; 3:902-907. [PubMed: 26286418]

33. Kumal RR, Landry CR, Abu-Laban M, Hayes DJ, Haber LH. Monitoring the Photocleaving Dynamics of Colloidal MicroRNA-Functionalized Gold Nanoparticles Using Second Harmonic Generation. Langmuir. 2015; 31:9983-9990. [PubMed: 26313536]

34. Chase HM, Psciuk BT, Strick BL, Thomson RJ, Batista VS, Geiger FM. Beyond Local Group Modes in Vibrational Sum Frequency Generation. J. Phys. Chem. A. 2015; 119:3407-3414. [PubMed: 25774902] 
35. Liu X, Biswas S, Jarrett JW, Poutrina E, Urbas A, Knappenberger KL, Vaia RA, Nealey PF. Deterministic Construction of Plasmonic Heterostructures in Well-Organized Arrays for Nanophotonic Materials. Adv. Mater. 2015; 27:7314-7319. [PubMed: 26463579]

36. Czaplicki R, Mäkitalo J, Siikanen R, Husu H, Lehtolahti J, Kuittinen M, Kauranen M. SecondHarmonic Generation from Metal Nanoparticles: Resonance Enhancement versus Particle Geometry. Nano Lett. 2015; 15:530-534. [PubMed: 25521745]

37. Capretti A, Pecora EF, Forestiere C, Dal Negro L, Miano G. Size-Dependent Second-Harmonic Generation From Gold Nanoparticles. Phys. Rev. B: Condens. Matter Mater. Phys. 2014; 89:125414.

38. Dadap JI, Eisenthal KB. Probing the Relative Orientation of Molecules Bound to DNA by SecondHarmonic Generation. J. Phys. Chem. B. 2014; 118:14366-14372. [PubMed: 25379958]

39. Kumal RR, Karam TE, Haber LH. Determination of the Surface Charge Density of Colloidal Gold Nanoparticles Using Second Harmonic Generation. J. Phys. Chem. C. 2015; 119:16200-16207.

40. Leone SR, Voth GA, Dai H-L. Experimental and Theoretical Probing of Molecular Dynamics at Catalytic and Ionic Liquid Interfaces. DTIC Document. 2014

41. Daniel J, Bondu F, Adamietz F, Blanchard-Desce M, Rodriguez V. Interfacial Organization in Dipolar Dye-Based Organic Nanoparticles Probed by Second-Harmonic Scattering. ACS Photonics. 2015; 2:1209-1216.

42. Doughty B, Ma Y-Z, Shaw RW. Probing Interfacial Electronic States in CdSe Quantum Dots Using Second Harmonic Generation Spectroscopy. J. Phys. Chem. C. 2015; 119:2752-2760.

43. Svechkarev D, Kolodezny D, Mosquera-Vázquez S, Vauthey E. Complementary Surface Second Harmonic Generation and Molecular Dynamics Investigation of the Orientation of Organic Dyes at a Liquid/Liquid Interface. Langmuir. 2014; 30:13869-13876. [PubMed: 25393042]

44. Karam TE, Haber LH. Molecular Adsorption and Resonance Coupling at the Colloidal Gold Nanoparticle Interface. J. Phys. Chem. C. 2014; 118:642-649.

45. Karam TE, Smith HT, Haber LH. Enhanced Photothermal Effects and Excited-State Dynamics of Plasmonic Size-Controlled Gold-Silver-Gold Core-Shell-Shell Nanoparticles. J. Phys. Chem. C. 2015; 119:18573-18580.

46. Haber LH, Kwok SJJ, Semeraro M, Eisenthal KB. Probing the Colloidal Gold Nanoparticle/ Aqueous Interface With Second Harmonic Generation. Chem. Phys. Lett. 2011; 507:11-14.

47. Haber LH, Eisenthal KB. Molecular Excited-State Relaxation Dynamics at the Colloidal Microparticle Interface Monitored with Pump-Probe Second Harmonic Generation. J. Phys. Chem. B. 2013; 117:4249-4253. [PubMed: 22928978]

48. Xu B, Gonella G, DeLacy BG, Dai H-L. Adsorption of Anionic Thiols on Silver Nanoparticles. J. Phys. Chem. C. 2015; 119:5454-5461.

49. Gan W, Gonella G, Zhang M, Dai H-L. Communication: Reactions and Adsorption at the Surface of Silver Nanoparticles Probed by Second Harmonic Generation. J. Chem. Phys. 2011; 134:041104. [PubMed: 21280679]

50. Shen, YR. The Principles of Nonlinear Optics. Hoboken, NJ, USA: Wiley; 2003.

51. Valev V. Characterization of Nanostructured Plasmonic Surfaces With Second Harmonic Generation. Langmuir. 2012; 28:15454-15471. [PubMed: 22889193]

52. Smirnova DA, Shadrivov IV, Miroshnichenko AE, Smirnov AI, Kivshar YS. Second-Harmonic Generation by a Graphene Nanoparticle. Phys. Rev. B: Condens. Matter Mater. Phys. 2014; 90:035412.

53. Roke S, Gonella G. Nonlinear Light Scattering and Spectroscopy of Particles and Droplets in Liquids. Annu. Rev. Phys. Chem. 2012; 63:353-378. [PubMed: 22263911]

54. Yan ECY, Liu Y, Eisenthal KB. New Method for Determination of Surface Potential of Microscopic Particles by Second Harmonic Generation. J. Phys. Chem. B. 1998; 102:6331-6336.

55. Darlington AM, Gibbs-Davis JM. Bimodal or Trimodal? The Influence of Starting pH on Site Identity and Distribution at the Low Salt Aqueous/Silica Interface. J. Phys. Chem. C. 2015; 119:16560-16567.

56. Campen RK, Pymer AK, Nihonyanagi S, Borguet E. Linking Surface Potential and Deprotonation in Nanoporous Silica: Second Harmonic Generation and Acid/Base Titration. J. Phys. Chem. C. 2010; 114:18465-18473. 
57. Schneider L, Peukert W. Second Harmonic Generation Spectroscopy as a Method for in Situ and Online Characterization of Particle Surface Properties. Part. Part. Syst. Charact. 2006; 23:351-359.

58. Walter SR, Geiger FM. DNA on Stage: Showcasing Oligonucleotides at Surfaces and Interfaces with Second Harmonic and Vibrational Sum Frequency Generation. J. Phys. Chem. Lett. 2010; 1:9-15.

59. Li H, Xia H, Wang D, Tao X. Simple Synthesis of Monodisperse, Quasi-Spherical, CitrateStabilized Silver Nanocrystals in Water. Langmuir. 2013; 29:5074-5079. [PubMed: 23578217]

60. Pereira M, Lai EP. Capillary Electrophoresis for the Characterization of Quantum Dots After NonSelective or Selective Bioconjugation With Antibodies for Immunoassay. J. Nanobiotechnol. 2008; 6:10.

61. Evans, DF; Wennerström, H. The Colloidal Domain: Where Physics, Chemistry, Biology, and Technology Meet. New York: WILEY-VCH; 1999.

62. Doughty B, Kazer SW, Eisenthal KB. Binding and Cleavage of DNA With the Restriction Enzyme EcoR1 Using Time-Resolved Second Harmonic Generation. Proc. Natl. Acad. Sci. U. S. A. 2011; 108:19979-19984. [PubMed: 22114185]

63. Karam TE, Khoury RA, Haber LH. Excited-State Dynamics of Size-Dependent Colloidal TiO2-Au Nanocomposites. J. Chem. Phys. 2016; 144:124704. [PubMed: 27036472] 

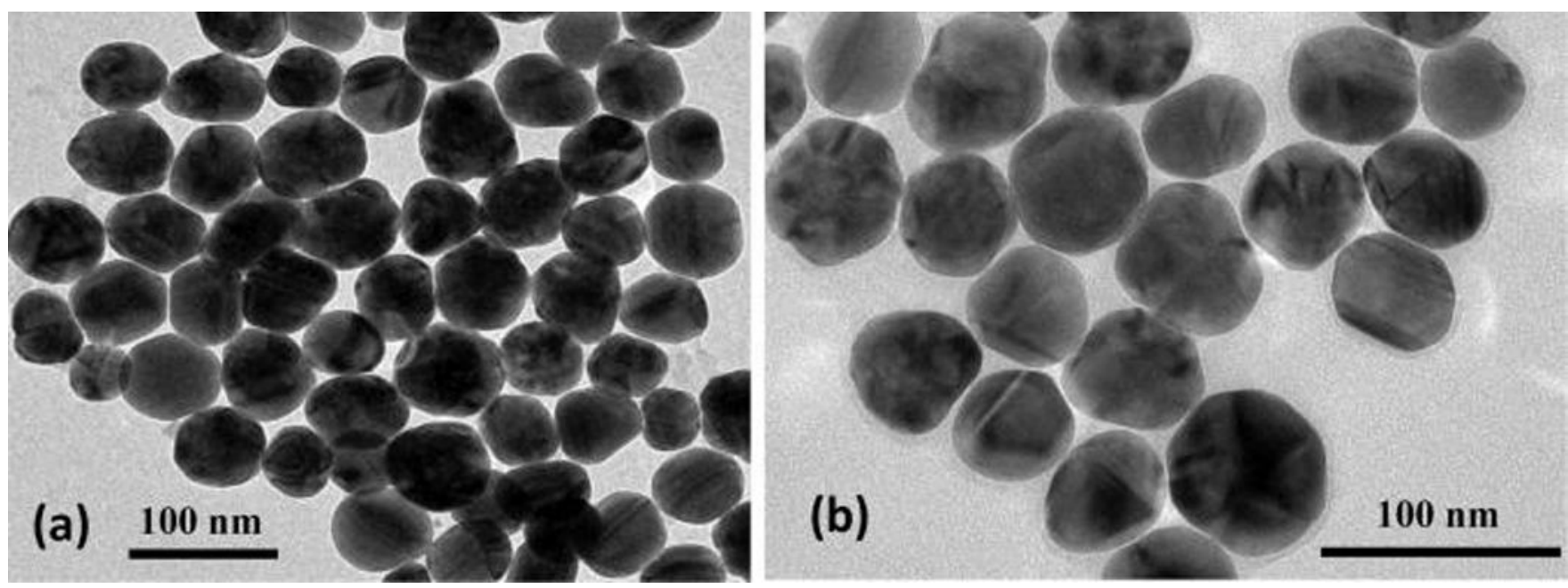

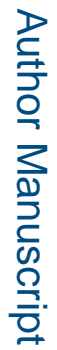

Figure 1.

Representative TEM images of (a) silver nanoparticles having size $65 \pm 7.5 \mathrm{~nm}$ and (b) miRNA-functionalized silver nanoparticles. 


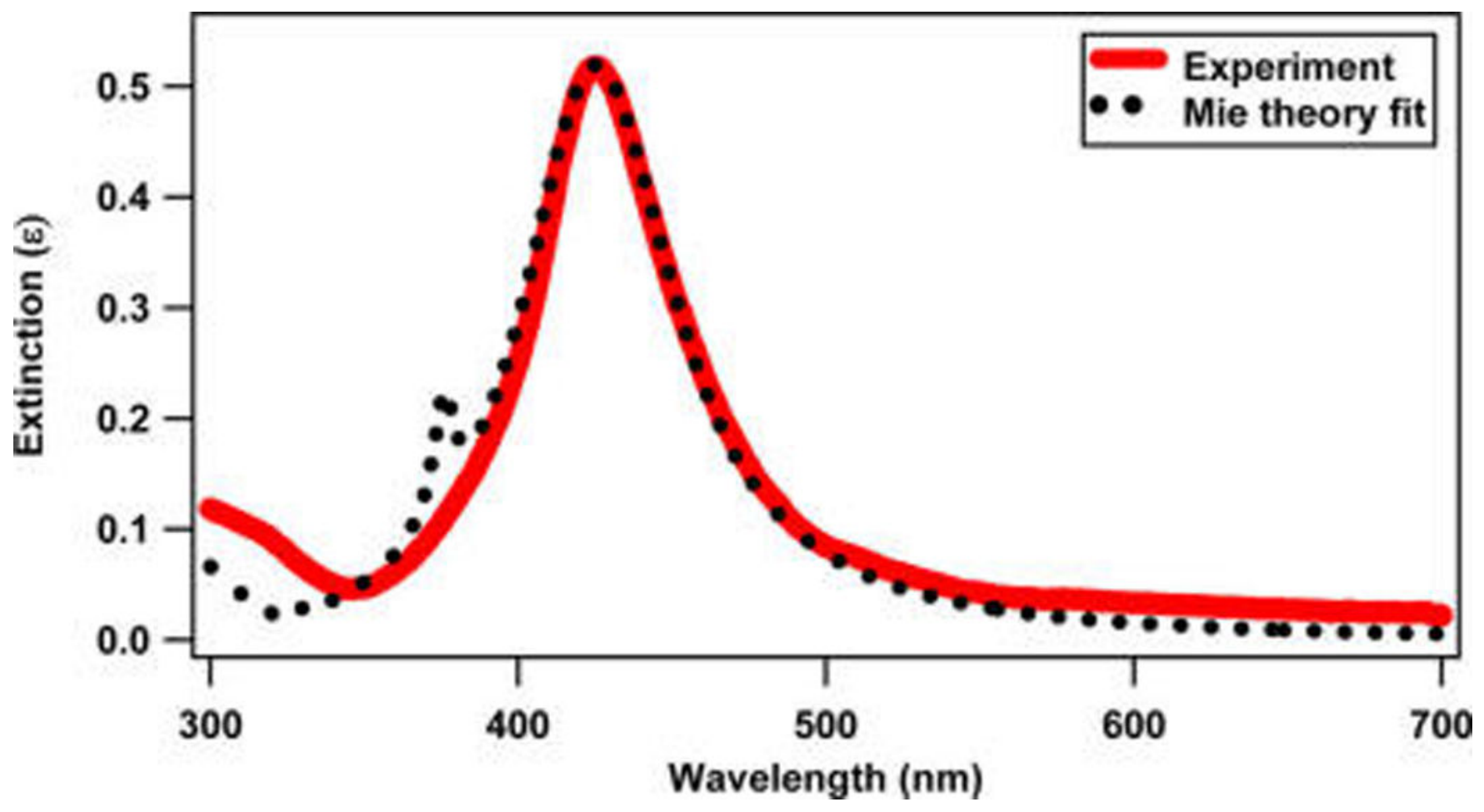

Figure 2.

Extinction spectra of $65 \pm 7.5 \mathrm{~nm}$ colloidal silver nanoparticles in water (red line) compared with Mie theory (dotted black line). 


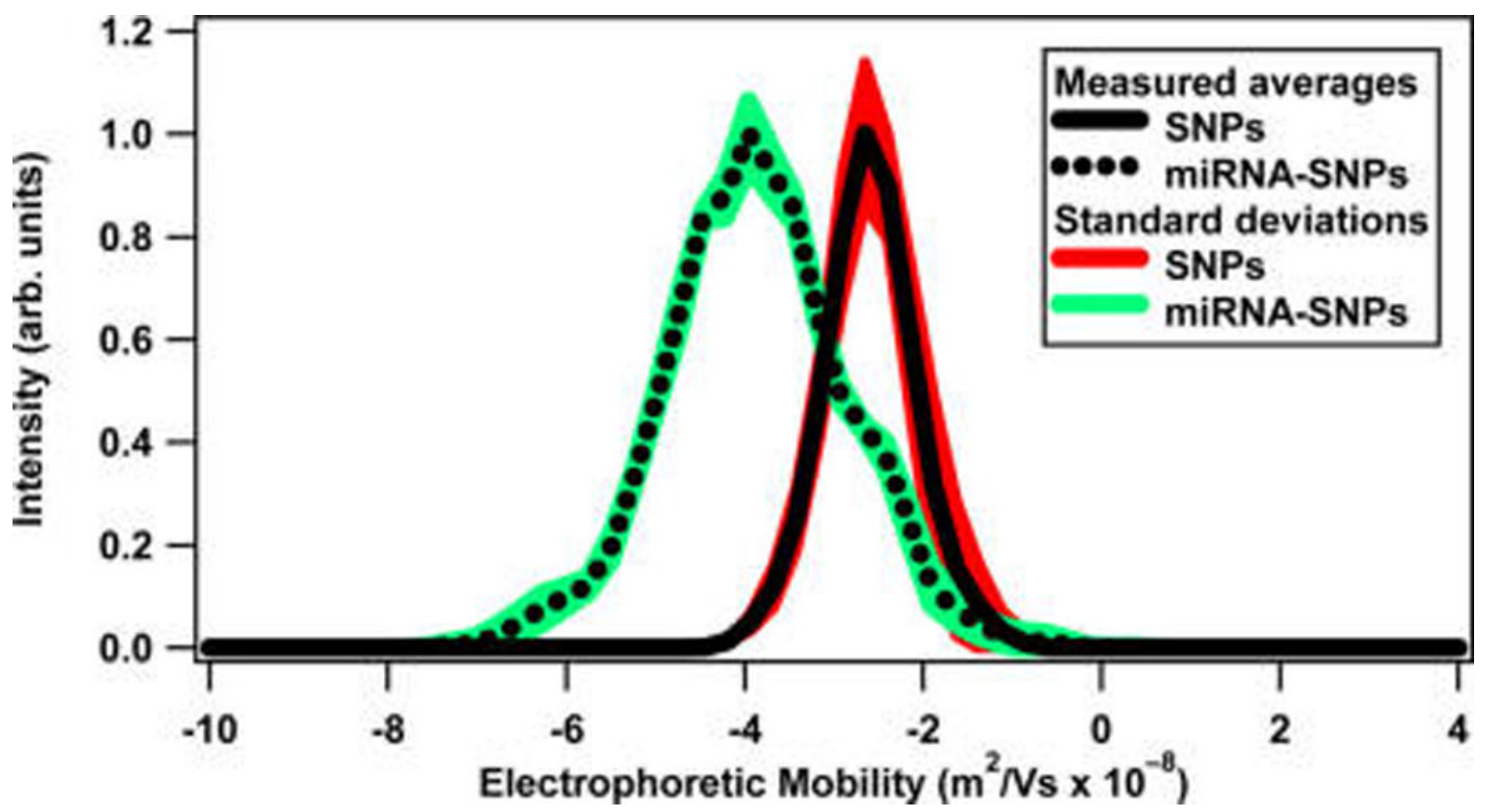

Figure 3.

Electrophoretic mobility plot of silver nanoparticles before and after miRNA functionalization showing the measured averages (solid and dotted black lines, respectively) and corresponding standard deviations (red and green areas, respectively). 

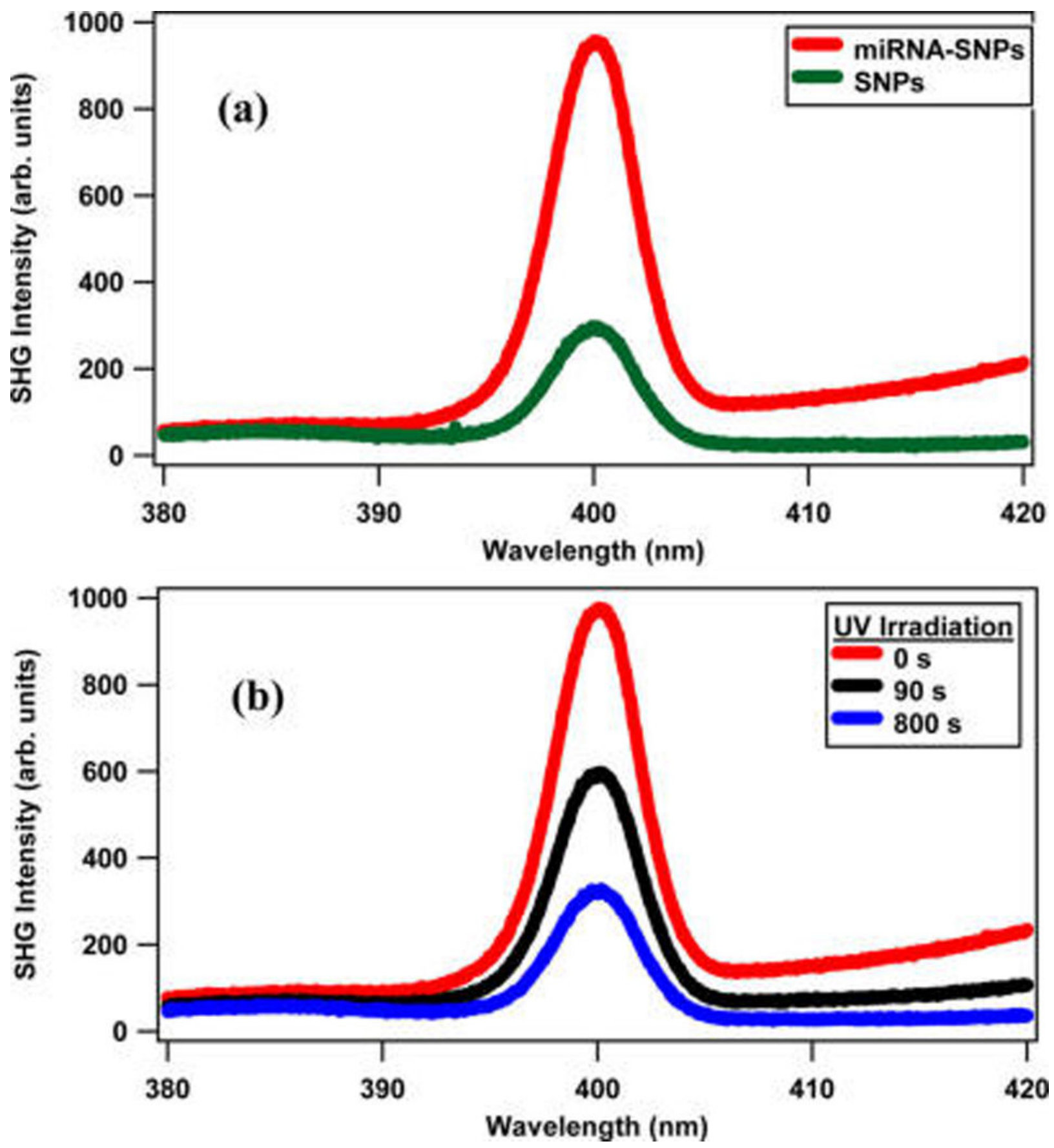

Figure 4.

(a) SHG spectra of the colloidal silver nanoparticles and the miRNA-functionalized silver nanoparticles. (b) SHG spectra of miRNA-functionalized colloidal silver nanoparticle sample after different UV irradiation times using $365 \mathrm{~nm}$ with $20 \mathrm{~mW}$ average power. The probe laser is fixed at $800 \mathrm{~nm}$. 

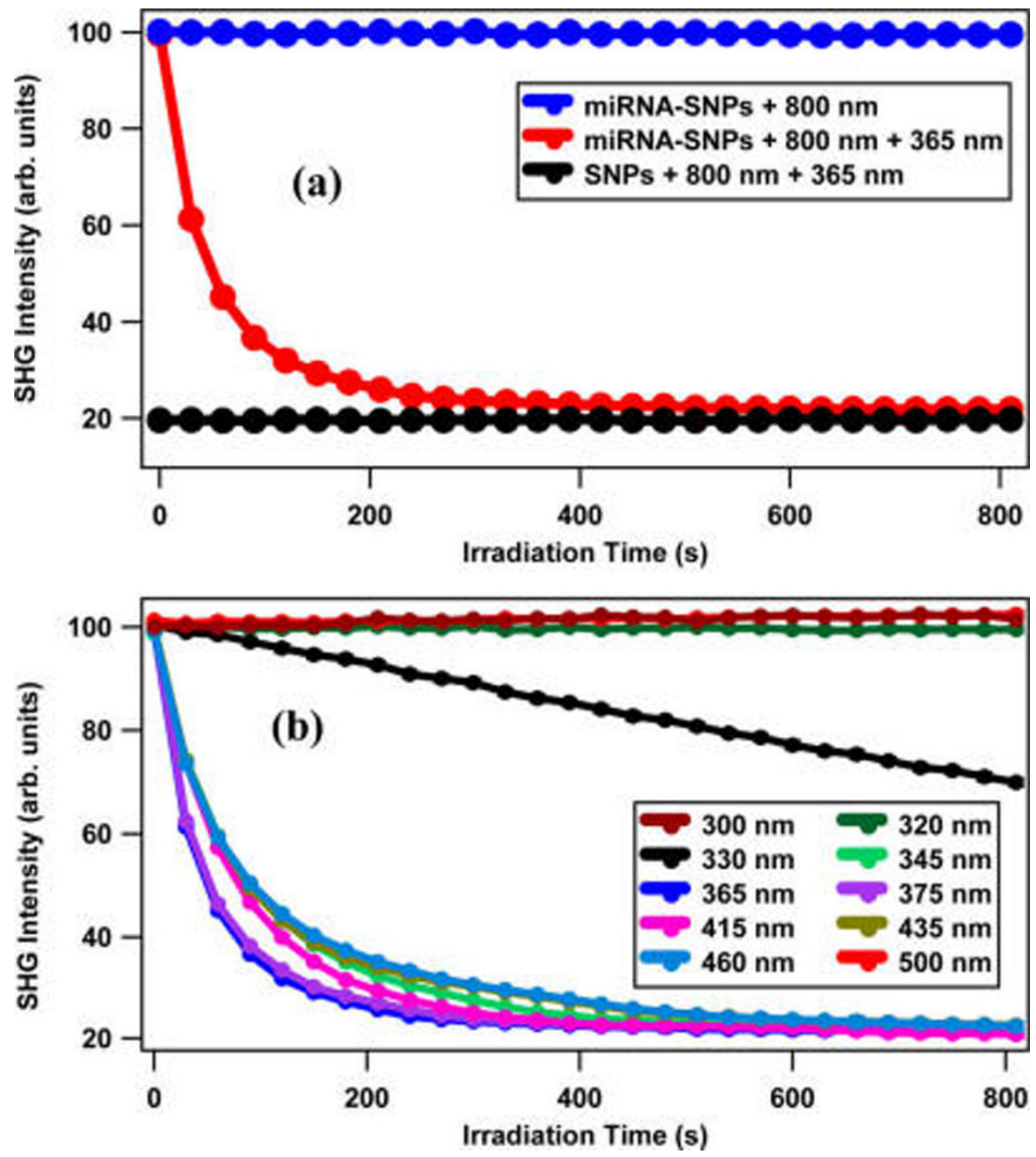

Figure 5.

(a) For control experiments, the time-dependent SHG signal remains constant for both the miRNA-functionalized SNPs using the $800 \mathrm{~nm}$ probe laser only (blue data) and the SNPs using the $800 \mathrm{~nm}$ probe and the UV laser at $365 \mathrm{~nm}$ (black data). The SHG of the miRNAfunctionalized SNPs decreases as a function of time under irradiation with the UV laser at $365 \mathrm{~nm}$ (red data). (b) Time-dependent SHG measurements of the miRNA-functionalized SNPs using different UV and visible laser wavelengths at $20 \mathrm{~mW}$ average power to determine the photocleaving dynamics. 


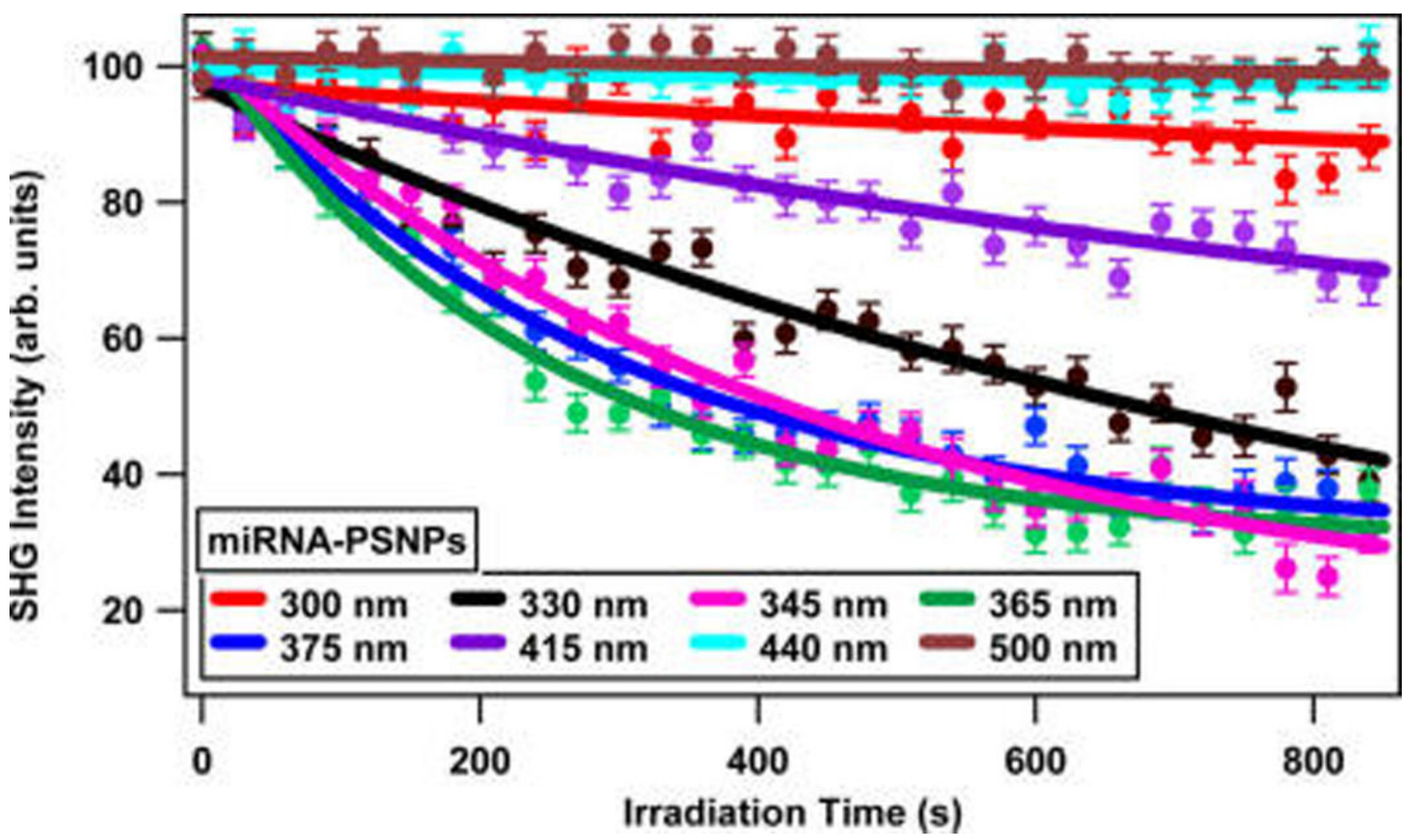

Figure 6.

Time-dependent SHG signals showing the photocleaving of miRNA-functionalized polystyrene nanoparticles using different irradiation wavelengths. 


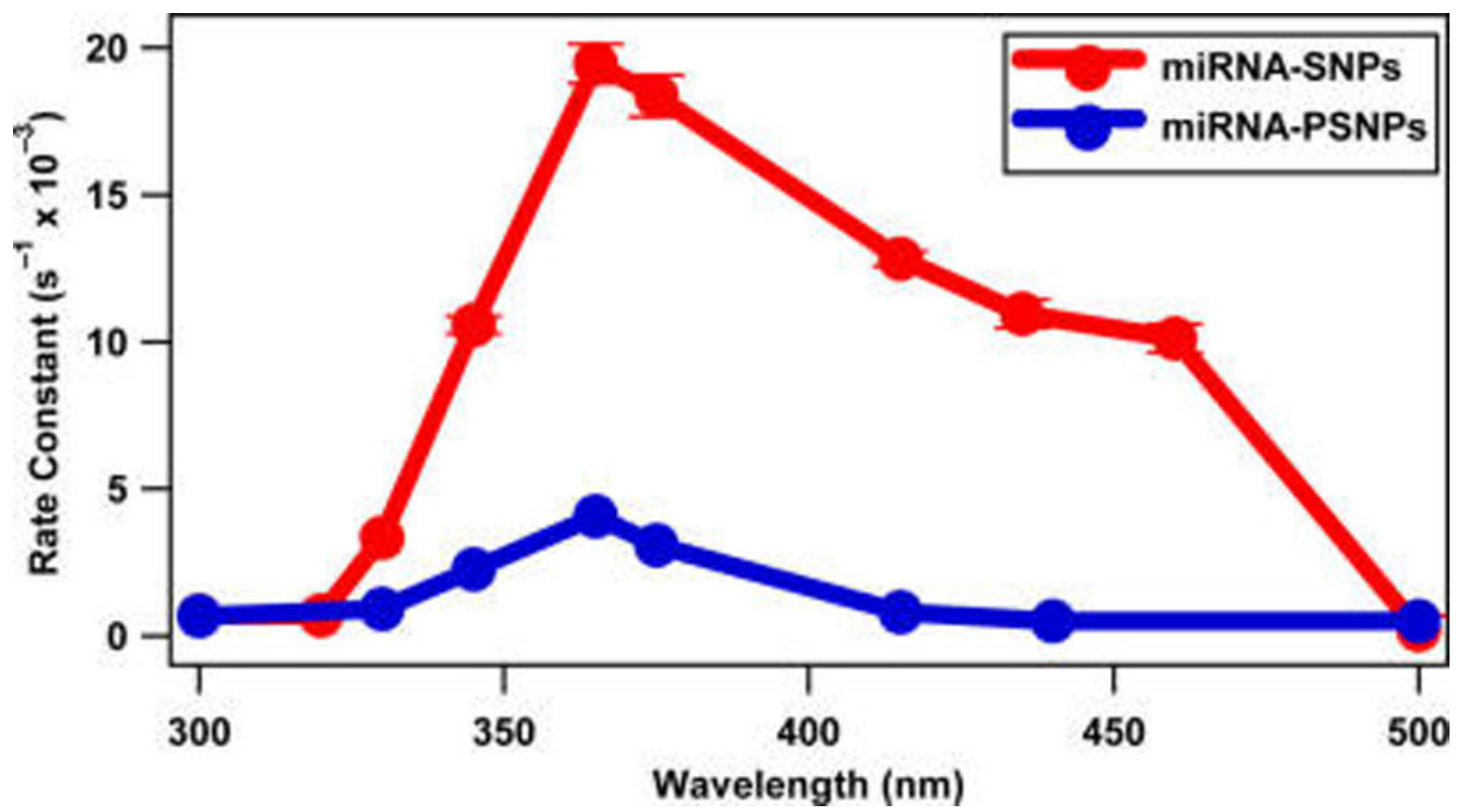

Figure 7.

Photocleaving rate constants of the miRNA-functionalized SNPs and PSNPs at different irradiation wavelengths using $20 \mathrm{~mW}$ average power. 

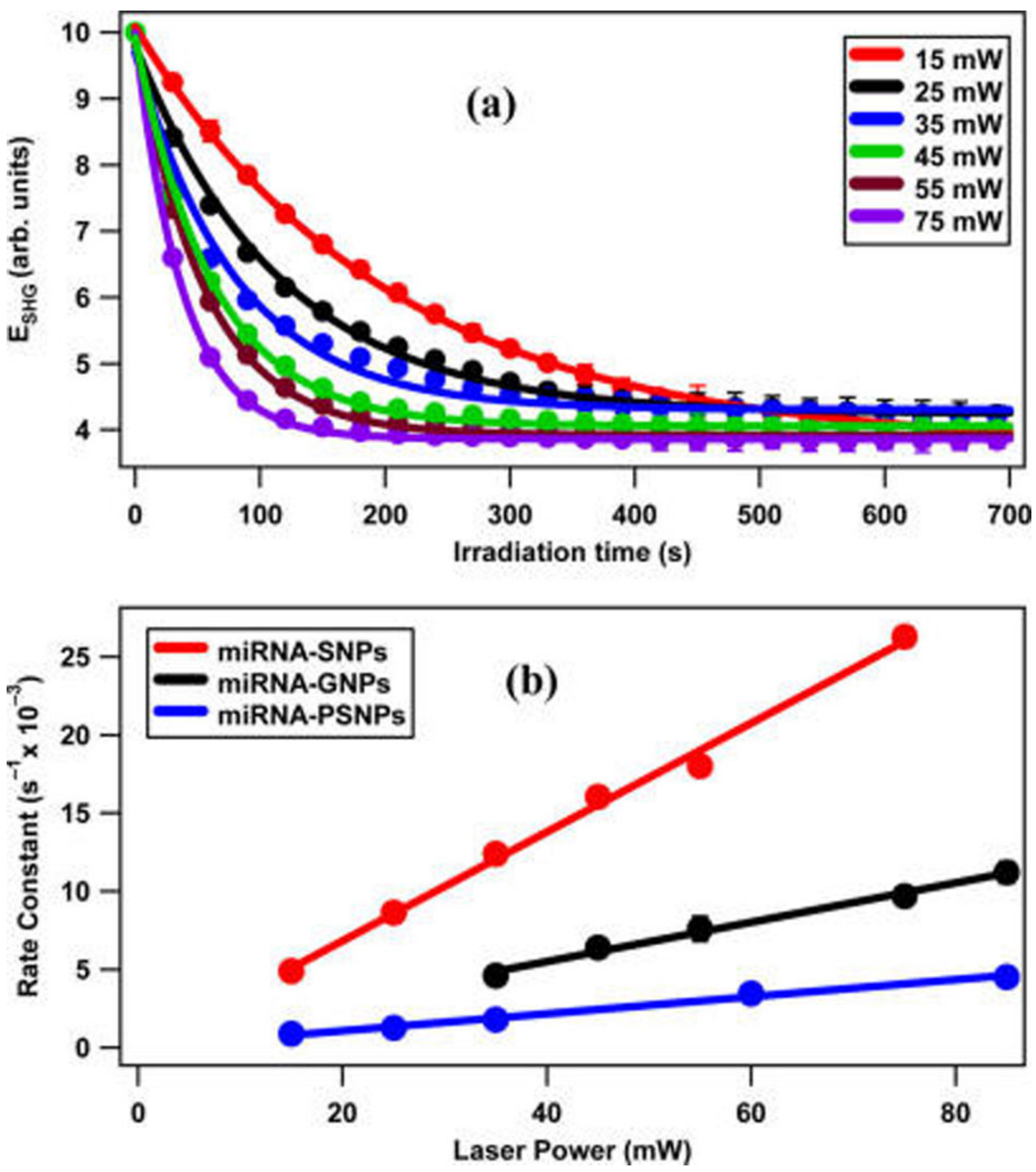

Figure 8.

(a) Measured SHG electric field from miRNA-functionalized SNPs as a function of irradiation time with $365 \mathrm{~nm}$ at different UV laser average powers with corresponding exponential fits. (b) The photocleaving rate constants for the miRNA-functionalized SNPs and PSNPs under varying UV laser powers. The linear variation with laser power indicates a one-photon photocleaving process for all nanoparticle samples. Previous results from GNPs are included for comparison. 


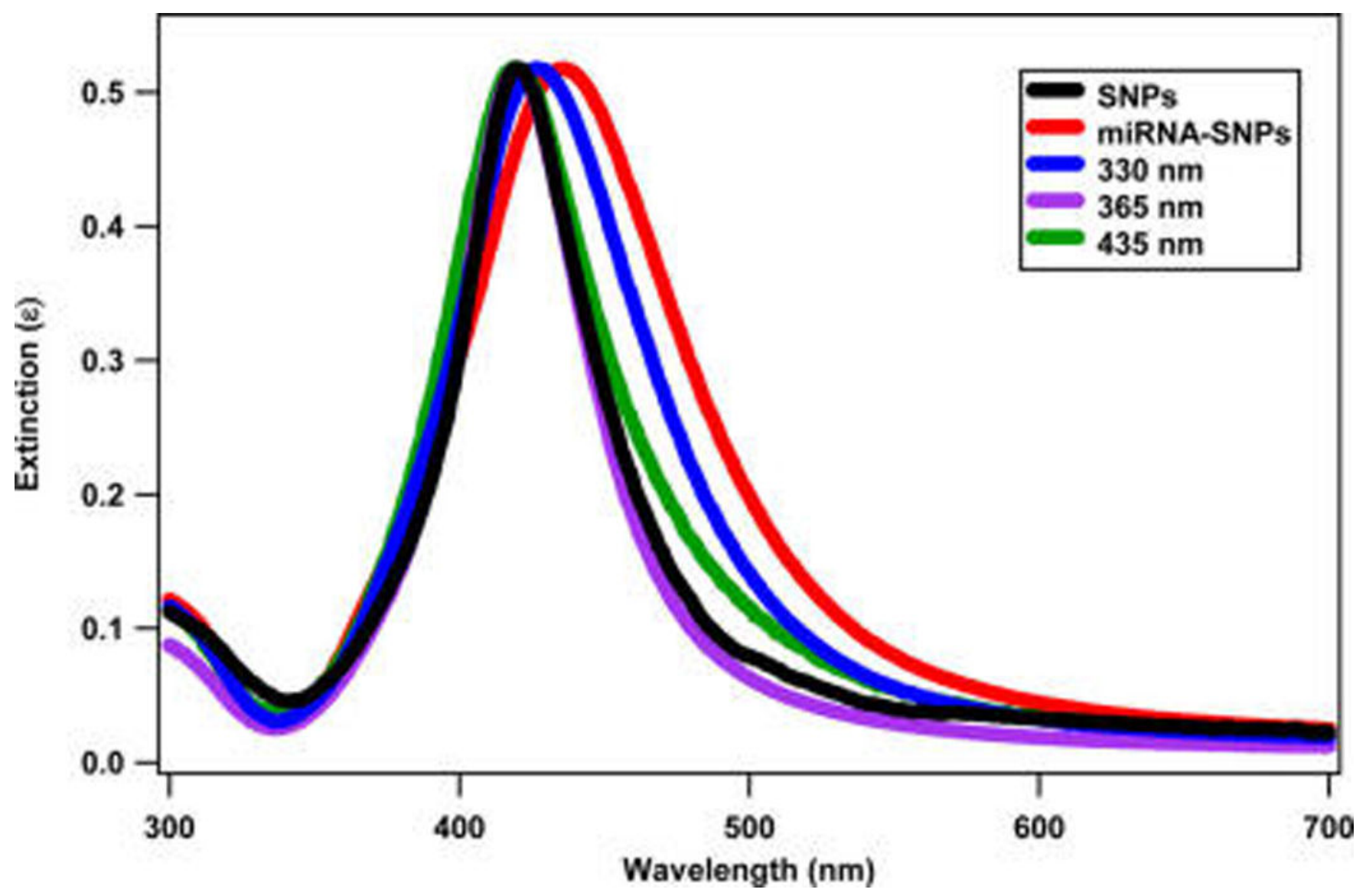

Figure 9.

Extinction spectra of the SNPs, the miRNA-functionalized SNPs, and the miRNAfunctionalized SNPs after laser irradiation with different wavelengths. 


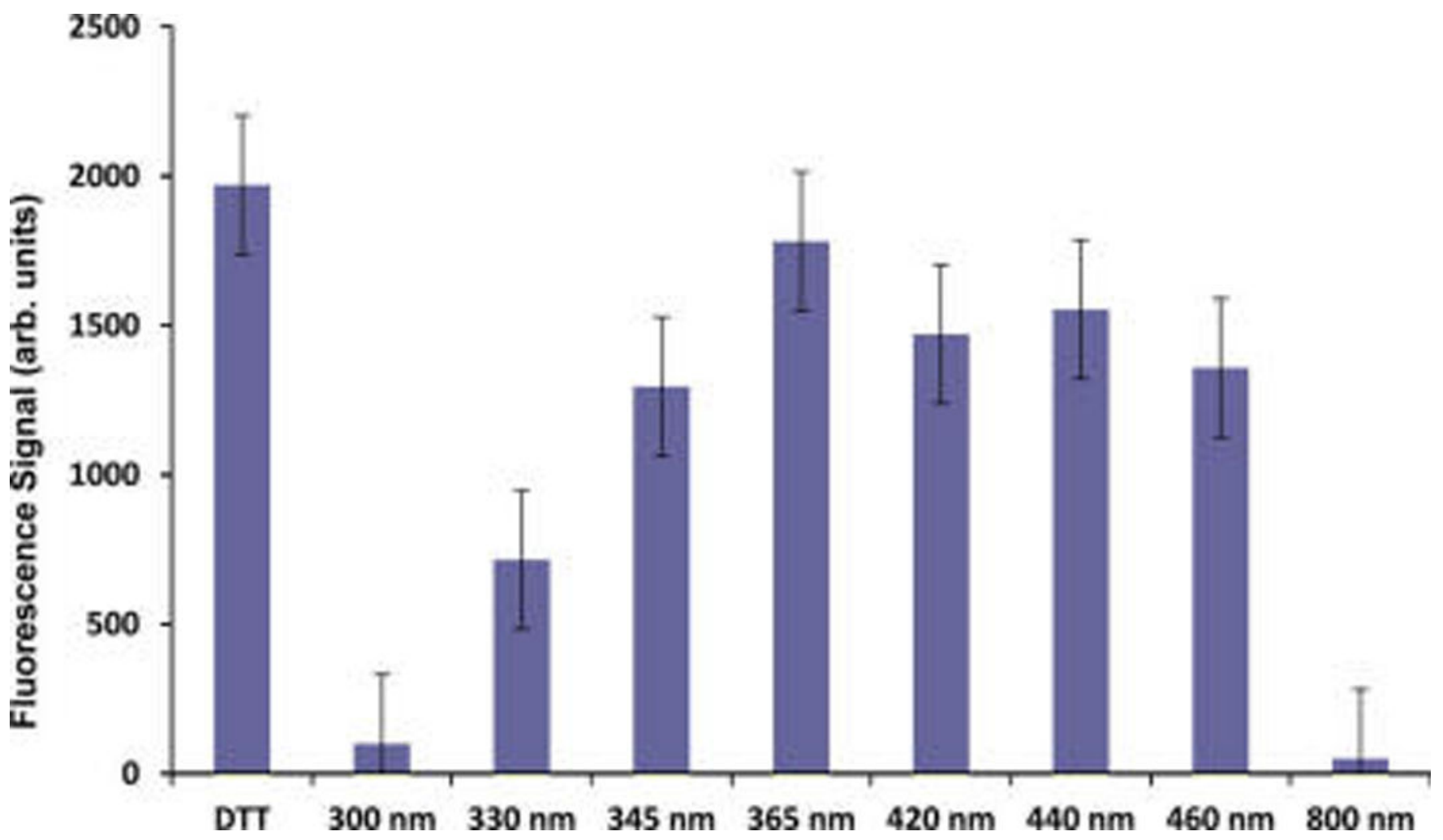

Figure 10.

Fluorescence measurements of miRNA-functionalized SNPs after DTT reduction and after using laser irradiation at different wavelengths varying from 300 to $800 \mathrm{~nm}$. 